\title{
VIVENCIANDO A SOBRECARGA AO VIR-A-SER UM CUIDADOR FAMILIAR DE PESSOA COM ACIDENTE VASCULAR CEREBRAL (AVC): UMA ANÁLISE DO CONHECIMENTO ${ }^{1}$
}

Bocchi SCM. Vivenciando a sobrecarga ao vir-a-ser um cuidador familiar de pessoa com acidente vascular cerebral (AVC): uma análise do conhecimento. Rev Latino-am Enfermagem 2004 janeiro-fevereiro; 12(1):115-21.

Trata-se de trabalho do tipo bibliográfico com a finalidade de fazer uma análise temática da produção do conhecimento em periódicos, acerca da sobrecarga em cuidadores familiares de pessoas com Acidente Vascular Cerebral (AVC). O corpus de análise reuniu artigos localizados nas décadas de 80 e 90, a partir das bases de dados Medline, Lilacs e Cinahl. A análise de conteúdo foi o referencial metodológico que permitiu organizar todo o conhecimento, em um corpo de categorias e subcategorias, denominadas: Categoria 1 - As seqüelas do AVC gerando sobrecarga; Categoria 2 - Aspectos gerando sobrecarga, congregando as subcategorias: o isolamento social, as mudanças e as insatisfações conjugais, as dificuldades financeiras e os déficits na saúde física e no autocuidado do cuidador; Categoria 3 - Outras análises relacionadas à sobrecarga em cuidadores familiares.

DESCRITORES: acidente cerebrovascular; efeitos psicossociais da doença; família

\section{LIVING THE BURDEN IN BECOMING A FAMILY CAREGIVER FOR A CEREBROVASCULAR ACCIDENT SURVIVOR: KNOWLEDGE ANALYSIS}

This bibliographical research aims to carry out a thematic analysis of knowledge production in periodicals, about the burden placed on family caregivers of Cerebrovascular Accident (CVA) survivors. The analysis gathered articles found in the 80 's and 90's, through the Medline, Lilacs and Cinahl databases. The content analysis was the methodological background that enabled us to organize the whole knowledge into a body of categories and subcategories, called: Category 1 - The sequels of the CVA creating burden, Category 2 - Aspects generating burden, covering the following subcategories: social isolation, marital changes and dissatisfactions, family caregivers' financial difficulties and deficits in their physical health and self-care, Category 3 - Other analyses about burden in CVA survivors' family caregivers.

DESCRIPTORS: cerebrovascular accident; psychosocial effects of the illness; family

\section{VIVIENDO LA SOBRECARGA AL CONVERTIRSE EN CUIDADOR FAMILIAR DE PERSONAS CON ACCIDENTE CEREBROVASCULAR: ANÁLISIS DEL CONOCIMIENTO}

Se trata de un trabajo del tipo bibliográfico con la finalidad de hacer un análisis temático de producción del conocimiento en revistas, sobre la carga de cuidadores familiares de personas con Accidente Cerebrovascular (ACV). El corpus de análisis reunió artículos realizados en las décadas de 80 y 90 a partir de las bases de datos Medline, Lilacs y Cinahl. El análisis de contenido fue el referencial metodológico que permitió organizar todo el conocimiento en un cuerpo de categorías y subcategorías denominadas: categoría 1 - Las secuelas del ACV generando carga, Categoría 2 - Aspectos generadores de la carga que congregan las subcategorías: el aislamiento social, los cambios y las insatisfacciones conyugales, las dificultades financieras y los déficit en la salud física y en el cuidado consigo mismo, Categoría 3 - Otros análisis sobre la carga de cuidadores familiares de personas con ACV.

DESCRIPTORES: accidente cerebrovascular; costo de enfermedad; familia

\footnotetext{
${ }^{1}$ Este artigo é parte de uma pesquisa mais extensa subsidiada pelo CNPq, enquanto cursando o Doutorado na Escola de Enfermagem da Universidade de São Paulo e participando das atividades junto ao Grupo de Estudos de Enfermagem da Família (GEENF), sob a coordenação da Prof ${ }^{a}$ Dr $^{\underline{a}}$ Margareth Angelo; ${ }^{2}$ Professor Assistente Doutor da Faculdade de Medicina de Botucatu da Universidade Estadual Paulista, e-mail: sbocchi@fmb.unesp.br
} 
INTRODUÇÃO

Este artigo é parte de um trabalho mais extenso, no qual estão sendo desenvolvido processos investigatórios sobre cuidadores familiares de pessoas com AVC, visando ampliar o conhecimento sobre o objeto, como estratégia retroalimentadora do ensino, da pesquisa e da assistência.

Num primeiro momento, foi realizado um estudo bibliográfico em periódicos, quando foi analisada a produção do conhecimento sobre a temática ${ }^{(1)}$.

O trabalho apontou ser um conhecimento, essencialmente internacional, tendo seu marco na década de 80, sendo recente, tanto para a Enfermagem, quanto para as outras profissões interessadas no trabalho com cuidadores familiares de pessoas com $\mathrm{AVC}^{(1)}$.

As tendências demonstraram haver aumento na produtividade de trabalhos científicos, sinalizando aprofundamento na temática para as próximas décadas, tanto em pólos tradicionais, representados pelos Estados Unidos e Reino Unido e países vizinhos, quanto expansibilidade para outros continentes ${ }^{(1)}$.

No Brasil, o conhecimento se encontra em processo de emergência, em grandes centros de pósgraduação, a partir da segunda metade da década de $90^{(1)}$.

Como o relatório final do trabalho se apresentou extenso e por se tratar de assunto pouco explorado em nosso país, em tempos em que o AVC vem se constituindo como causa principal de internações, mortalidade e incapacidades e, justamente, quando o Programa de Saúde da Família (PSF) é operacionalizado, o que tem remetido a Enfermagem à busca de referenciais norteadores de sua assistência junto às famílias, é que foi objetivada a comunicação do conhecimento, por meio de vários artigos.

Neste artigo, está sendo apresentado o conhecimento relacionado à sobrecarga vivenciada pelos cuidadores familiares de pessoas com AVC, que se constituiu em uma das temáticas relevantes a partir dos artigos analisados.

Ao emergir a temática "vivenciando a sobrecarga", houve a necessidade de estender, explorando o seu significado, por se tratar de nomenclatura intimamente relacionada aos efeitos negativos das incapacidades geradas pelo AVC e ao papel assumido pelo cuidador familiar.

Pesquisando autores que exploram a questão conceitual da sobrecarga da doença sobre famílias, verificou-se que não se trata de conceito recente, sendo utilizado desde 1946, referindo-se às conseqüências naqueles que estão em contato próximo com um paciente psiquiátrico, severamente perturbado. A questão tem se relacionado às interações em longo prazo, ao cuidar de pacientes com distúrbios mentais ${ }^{(2)}$.

Este conceito é denominado pela literatura inglesa por meio da nomenclatura burden, decorrente da introdução da filosofia da psiquiatria social nas décadas de 50 e 60 , períodos em que se começou a dar ênfase às discussões das vantagens e sobrecargas acumuladas nas famílias de pacientes tratados no domicílio e, mais recentemente, com o aumento do interesse pela unidade familiar como sujeito de cuidado ${ }^{(2)}$.

Platt, em 1985, foi o primeiro estudioso a apresentar uma definição elaborada acerca dessa sobrecarga, relacionado-a à presença de problemas, dificuldades ou eventos adversos que afetam as vidas dos familiares $^{(3)}$.

As pesquisas conduzidas sobre os eventos da vida e o suporte social dividem a sobrecarga familiar em objetiva e subjetiva.

A sobrecarga objetiva engloba os sintomas e comportamentos de pacientes psiquiátricos dentro de seus ambientes sociais e suas conseqüências, enquanto a sobrecarga subjetiva está relacionada às conseqüências psicológicas para a família ${ }^{(2)}$.

Pesquisando diversos estudos, há aquele que consegue levantar oito aspectos relacionados à sobrecarga e à doença mental, sendo os primeiros seis considerados objetivos, enquanto os dois últimos subjetivos. São eles: rotina familiar, relações familiares, relações sociais, lazer e profissão, finanças, crianças e parentes, saúde, estresse, incluindo, a esse último, sentimentos e emoções negativas geradas pela experiência ${ }^{(2)}$.

\section{MÉTODO}

Este estudo se constitui num recorte, reunindo 18 artigos, a partir de pesquisa bibliográfica com 70 artigos, localizados nas bases de dados: Medline, Lilacs e Cinahl, tomando-se o cuidado de rastreá-los desde o início das mesmas até dezembro de 2000 , tendo como palavraschave e combinações as palavras "Cerebrovascular Accident and carer or caregiver or spouse or partner" e 
restritos às línguas Portuguesa, Espanhola e Inglesa.

A análise de conteúdo foi o referencial teórico utilizado para se analisar os dados ${ }^{(4)}$.

A análise se desdobrou em três fases: préanálise, exploração do material e interpretação.

a) Pré-análise: nesta fase realizou-se uma leitura flutuante e a versão dos artigos para o português, procurando verificar se realmente os trabalhos tratavam do objeto a ser explorado (cuidadores familiares de pessoas com AVC).

b) Exploração do material: ocorreu logo após a préanálise e consistiu numa fase de operações de codificação, classificação e agregação, em função dos significados. Nesta fase, o material foi codificado, ou seja, submetido a "um processo pelo qual os dados brutos são transformados sistematicamente e agregados em unidades, as quais permitem uma descrição exata das características pertinentes do conteúdo"(6). Para organização realizou-se: - o recorte (escolha das unidades de significação); - a classificação e agregação (categorização). Para proceder ao recorte do material, tornou-se necessária a leitura do mesmo e a demarcação dos "núcleos de sentido", ou seja, das unidades de significação. Essas unidades podem ser chamadas de unidades de registro, que nada mais são do que um segmento de conteúdo a ser considerado como unidade de base, visando a categorização. No caso de uma análise temática, o tema é a unidade de significação, que se libera naturalmente de um texto analisado. Logo, fazer uma análise temática consiste em descobrir os temas, que são as unidades de registro nesse tipo de análise e que corresponde a uma regra para o recorte. Após o recorte, as unidades de significação foram classificadas e agregadas em categorias e subcategorias.

c) Tratamento dos resultados obtidos, a inferência e a interpretação: nesta fase optou-se por trabalhar na linha qualitativa, por meio de significados, ao invés de inferências estatísticas, conforme preconizado por Minayo $^{(5)}$.

Dentre o corpo de categorias e subcategorias levantadas no trabalho, apresenta-se, neste artigo, a produção do conhecimento relativa apenas à temática "vivenciando a sobrecarga", reunindo três categorias, sendo elas: 1 - as seqüelas do AVC gerando a sobrecarga; 2 aspectos gerando a sobrecarga, congregando as subcategorias: isolamento social, mudanças e insatisfações conjugais, dificuldades financeiras e déficits na saúde física e no autocuidado; 3 - outras análises relacionadas à sobrecarga de cuidadores familiares.

Diante do exposto, será apresentado a produção do conhecimento de forma sistematizada.

\section{APRESENTANDO A PRODUÇÃO DO CONHECIMENTO}

As seqüelas do AVC gerando sobrecarga

Os déficits funcional e cognitivo, mudança de personalidade ou comportamental ${ }^{(6-7)}$, bem como da comunicação $^{(8)}$, são alterações impostas pelo AVC. Elas geram níveis de incapacidades, comprometendo não somente o paciente, mas a família e a comunidade ${ }^{(9)}$.

Os graus de incapacidades do paciente determinam os níveis de dependência por assistência e, conseqüentemente, um desafio ao cuidador familiar.

Dessa forma, surgem seis pesquisas na década de 90, explorando as relações entre as variáveis dependência e sobrecarga.

A sobrecarga está, freqüentemente, associada ao nível de dependência física ${ }^{(10-11)}$. Esse achado é corroborado por outro estudo, acrescentando que o acometimento da deambulação é fator importante na qualidade de vida dos cônjuges. A análise estatística desta pesquisa mostra correlação significativa entre a qualidade de vida do cuidador e a situação funcional do paciente por meio de instrumentos que permitem esse tipo de avaliações do doente ${ }^{(12)}$. No entanto, há outra pesquisa que, ao avaliar os problemas vivenciados por cuidadores familiares de pessoas com AVC, após a alta hospitalar, observou que, embora os cuidadores reconhecessem as mudanças emocionais, cognitivas e funcionais nos doentes, o que resultou em maior sobrecarga durante a recuperação foi a mudança comportamental ${ }^{(13)}$.

Além disso, há estudo no qual os autores referem não ter encontrado relacionamento significante entre doença emocional em cuidadores e o grau de incapacidade do paciente ${ }^{(14)}$, bem como outro evidenciando que os cuidadores de pacientes, severamente deficientes, tinham mais sobrecarga, porém essa não parecia estar relacionada aos níveis de dependência dos mesmos e, sim, ao tempo que o cuidador estava oferecendo assistência a uma pessoa dependente. No entanto, os resultados dessa pesquisa sugerem que a sobrecarga 
emocional é comum entre cuidadores de pacientes que sofreram um AVC e que estão debilitados física e emocionalmente. Assim, os autores acreditam que o estado emocional é provavelmente dependente de outros fatores, tais como: a personalidade e o ambiente, nos quais eles estão cuidando e que não foram avaliados pelo estudo $^{(15)}$.

\section{Aspectos gerando sobrecarga}

O isolamento social

Os cuidadores de pessoas com AVC vivenciam mudanças no estilo de vida ${ }^{(16)}$ que reduzem ${ }^{(17)}$, modificam $^{(12)}$ e geram insatisfações na vida social dos mesmos $^{(18)}$, devido às condições limitantes impostas pela doença, trazendo sentimentos de isolamento ${ }^{(19)} \mathrm{e}$ proporcionado relacionamento mais próximo e circunscrito às atividades domésticas ${ }^{(20)}$.

Os fatores limitantes da vida social do cuidador são mostrados a seguir:

- A sobrecarga de atividades. O acúmulo de atividades de trabalho com as tarefas de casa ${ }^{(12)}$ faz com que os cuidadores sintam-se ocupados ${ }^{(6)}$, experienciando limitação do tempo livre, como conseqüência direta por estarem assumindo o cuidado do ente ${ }^{(14,16)}$.

\section{- A própria perda do companheiro em atividades sociais $^{(6)}$.}

- Os distúrbios comportamentais do portador de AVC.

A apatia, a indiferença, a falta de motivação, a irritabilidade e a própria dependência do paciente levam os cuidadores a se recusarem em sair sozinhos ou a impor limites no período de passeio, gerando insatisfação antecipada ao evento, promovendo o confinamento e as alterações comportamentais $^{(14,21)}$.

\section{- As mudanças nos relacionamentos familiares e no} círculo de amizades. Nas relações familiares podem ocorrer o aumento dos conflitos ou maior aproximação entre os membros ${ }^{(16)}$. Os laços com parentes mais próximos, geralmente, são preservados e reforçados após o AVC, mas mesmo assim os cônjuges se queixam das limitações em encontrarem outros parentes ou $\operatorname{amigos}^{(12,20)}$, bem como vivenciam a redução de visitas de amigos com o passar do tempo ${ }^{(19)}$.

- A relutância por suporte. Muitos cuidadores reclamam da pouca ajuda prática da família e amigos e qualquer outra opção extensiva. Esse fator, no entanto, parece estar relacionado mais pela relutância do cuidador em solicitar ou aceitar ajuda, mantendo-se independente ${ }^{(22)}$. Isso parece ser um enigma quando os cuidadores, geralmente, são resistentes até mesmo em aceitarem o auxílio de serviços de cuidado-de-folga, quando disponíveis ${ }^{(16,23)}$. Essa atitude pode estar relacionada a várias causas como: ao medo de que esse ato representaria sinal de fracasso ou inadequação; ao medo e à ansiedade em abandonar o paciente na casa, sem atendimento, tornando-o suscetível ao agravamento das incapacidades; por eles estarem em risco de morte eminente; ou ainda visando a superproteção e excesso de cuidados, numa tentativa de amenizar os sentimentos de culpa pelas suas ações ou negligências ${ }^{(14)}$. Conseqüentemente, muitos cuidadores acabam expressando sentimentos de isolamento e/ou exaustão física $^{(14,20)}$, apoiados em justificativas de não poderem deixar os seus pacientes sozinhos ${ }^{(20)}$ ou em respeito ao próprio desejo do ente não querer permanecer só ${ }^{(12)}$.

- Isolamento pela nova vizinhança. Isso se dá, geralmente, quando é preciso mudar de casa para atender as necessidades do paciente ${ }^{(12)}$. Esses fatores acabam levando os cuidadores a se queixarem das poucas oportunidades de relaxar e gozar dos prazeres da vida ${ }^{(6)}$, tendo suas atividades de lazer reduzidas ou suprimidas ${ }^{(16)}$. Dentre elas, o comprometimento de viagens e férias ${ }^{(19)}$, mudanças nas atividades físicas e nas participações sociais comunitárias, sendo o atendimento religioso o que mais sentem falta ${ }^{(16)}$.

As mudanças e insatisfações conjugais

As relações conjugais têm se constituído numa das preocupações entre os cônjuges de pessoas com $\mathrm{AVC}^{(12)}$. Eles relatam estar vivenciando mudanças radicais impostas pela doença ${ }^{(18)}$, levando-os a se sentirem insatisfeitos com seus casamentos ${ }^{(8)}$.

Os papéis de cuidadores, freqüentemente, são modificados dentro do casamento, possibilitando novas experiências junto com seus parceiros, os quais são referidos como "não sendo mais as mesmas pessoas", mas uma outra, com seqüelas e todas as conseqüências geradas pela doença $^{(12)}$.

Os cônjuges acabam vivenciando os mesmos problemas do paciente, tais como: mudança no estilo de vida, depressão, frustração, trabalho extra, problemas de comunicação, perda da independência, da confiança e da concentração, sentimentos de inutilidade e de solidão ${ }^{(17)}$. 
Esse fenômeno é corroborado por outros autores, quando evidenciaram que os pacientes com níveis altos de irritabilidade e de depressão eram mais prováveis a ter esposas deprimidas ${ }^{(18)}$.

Um dos problemas na área da comunicação que pode intensificar a desarmonia nas relações conjugais é a presença de afasia ${ }^{(8,11)}$, gerando dificuldades na comunicação entre os cônjuges ${ }^{(18)}$, bem como podendo levar ao fim das relações sexuais ${ }^{(11)}$. O outro problema, nessa mesma área, é o relacionamento silencioso entre os casais, que tendem a ser tensos e se agravarem quando o casal permanece maior tempo sozinho durante a semana ${ }^{(18)}$.

Há dados demonstrando que $85 \%$ dos cônjuges não costumam ter relações sexuais desde a ocorrência do $\mathrm{AVC}^{(12)}$, além de ser caracterizado como assunto difícil de ser discutido entre os parceiros ${ }^{(19)}$.

Outro fator que, geralmente, pode agravar o relacionamento conjugal se dá quando um cônjuge deixa seu trabalho para cuidar do outro. O papel assumido tende a gerar pouca satisfação, desencadeando sentimentos de culpa por pensarem assim. Esses cuidadores tendem a compensá-los por meio da superproteção do doente ${ }^{(18)}$.

Existem, ainda, dois fatores intervenientes no processo de adaptação à nova situação. A primeira é a forma como o casal se relacionava anteriormente ao $\mathrm{AVC}^{(12)}$ e a segunda é o tempo de casado. O casal recémcasado tende a vivenciar conflitos em seus relacionamentos com respeito às limitações impostas pela doença. Eles, geralmente, encontram-se paralisados no processo de adaptação, solicitando aconselhamento conjugal, enquanto os casados há mais tempo expressam um processo de aceitação maior. No entanto, os autores evidenciaram um ponto positivo, que tanto um como o outro casal buscavam um novo equilíbrio, numa tentativa de resolver os efeitos do impacto da doença ${ }^{(19)}$.

As dificuldades financeiras

Os familiares cuidadores de pessoas com AVC geralmente têm problemas com o emprego ${ }^{(17)}$, abandonando ${ }^{(17-19)}$, reduzindo a jornada de trabalho ${ }^{(19-23)}$ ou tendo que sair mais cedo ${ }^{(19)}$ para se dedicarem ao cuidado da pessoa com $\operatorname{AVC}^{(11,18,23)}$. Estima-se que mais de $14 \%$ dos cuidadores desistem de seus empregos ${ }^{(11)}$.

Os efeitos do AVC sobre os empregos representam perdas e problemas financeiros ${ }^{(11,17-18)}$, mesmo recebendo os benefícios ${ }^{(17)}$.

Estudo aponta que o problema financeiro é uma das principais preocupações que os casais têm para atender as despesas com o portador de AVC, como parte de seus orçamentos domésticos. Alguns casais chegam a revelar a impossibilidade em contar com serviços de cuidado domiciliar ou efetuar reformas na casa para diminuir suas sobrecargas, por não terem recursos financeiros suficientes $^{(12)}$.

Os déficits na saúde física e no autocuidado do cuidador

$\mathrm{Na}$ tentativa de se explorar as necessidades e o bem-estar dos cuidadores, alguns trabalhos levantam alguns problemas como: cansaço entre a maioria dos cuidadores, distúrbios do sono pelo fato de dormirem tarde e terem seus sonos interrompidos para oferecerem cuidados $^{(23-24)}$, aparecimento de cefaléias ${ }^{(23)}$, perda de peso $^{(19-23)}$, sendo $25 \%$ deles hipertensos ${ }^{(24)}$. Outras alterações na saúde são levantadas junto a cuidadores familiares, quando esses se referem que, após assumirem o papel, perceberam mudanças como: ingestão maior de alimentos do que o habitual devido aos seus sentimentos, epigastralgias e cansaço para comer ${ }^{(16)}$.

Os cônjuges de pessoas com AVC relatam suas experiências com o aumento de trabalho em atividades como: cozinhar, dirigir e auxiliar os pacientes em atividades de higiene e de deambulação, respeitando as habilidades motoras do mesmo que, geralmente, estão comprometidas ${ }^{(19)}$. Isso os leva a ter um tempo limitado para se autocuidarem, bem como citam as mudanças financeiras, restringindo algumas possibilidades de terem uma melhor qualidade de vida ${ }^{(16)}$.

Há autores que, ao coletarem os dados, depararam com uma reação de surpresa pelos cônjuges quando eram questionados de que forma o AVC os afetava fisicamente. Isso parece ocorrer devido ao nível de envolvimento nos cuidados, levando-os a não prestar atenção nas suas próprias necessidades pessoais. Assim, os problemas de natureza emocional e física podem ocorrer devido à inobservância às necessidades de autocuidado ${ }^{(19)}$.

Outras análises relacionadas à sobrecarga de cuidadores familiares

As pesquisas que exploram a sobrecarga em familiares cuidadores de pessoas com AVC relatam que 
a mesma está associada a riscos de ruptura da integridade familiar e da qualidade de vida, tanto sob o aspecto familiar como do paciente ${ }^{(14)}$.

Em promessa de desempenharem o papel de cuidadores, os familiares acabam por experienciar a sobrecarga que, se excessiva, pode levá-los ao fracasso ou interrupção no suporte oferecido aos pacientes ${ }^{(14)}$.

Ao avaliar a qualidade de vida de familiares cuidadores de pessoas com AVC, estudiosos categorizaram os fatores adversos relatados pelos sujeitos em sobrecarga física e psicológica. A sobrecarga física estava mais relacionada aos pacientes mais dependentes, principalmente no transporte e na deambulação, visto que os serviços de atendimento domiciliares, geralmente ofereciam ajuda no banhar e no vestir-se. Além do que o cuidado consome tempo o que os levou a se queixarem de não poderem cuidar da própria saúde ${ }^{(12)}$.

A sobrecarga psicológica, também chamada de emocional, nem sempre é expressa, espontaneamente, pelos cônjuges, em parte pelos sentimentos de culpa, mas também por causa da atenção que, geralmente, está voltada para o paciente. Nesta pesquisa poucos se encontravam depressivos, mas todos se queixavam de estresse em excesso e dificuldades de coping às situações novas e às mudanças físicas e psicológicas demonstradas pelos pacientes. Eles expressavam ansiedade sobre 0 futuro e apreensão com outros problemas de saúde que pudessem surgir no paciente ou neles próprios ${ }^{(12)}$.

Esses autores verificaram que, do total de cuidadores familiares de pessoas com AVC avaliados, a maioria apresentava efeitos adversos sobre a saúde emocional, relacionados ao isolamento social (redução das atividades sociais e de lazer), sendo que a metade deles relatava efeitos adversos sobre o relacionamento familiar. Acrescentam ainda que $55 \%$ dos sujeitos investigados demonstravam evidências de sobrecarga emocional, principalmente quando cuidando de pacientes com demência ou distúrbios do comportamento.

Ademais, há pesquisa que, ao avaliar a relação entre as variáveis sobrecarga do cuidador e a morbidade psicológica, conclui que os aspectos psiquiátricos da doença crônica são mais estressores aos cuidadores que os aspectos físicos. Dentre os problemas encontrados estão os distúrbios comportamentais (apatia, agitação, perambular) e os distúrbios do humor (depressão, ansiedade) ${ }^{(25)}$.

Desta maneira, as avaliações clínicas e intervenções direcionadas para aqueles que cuidam de pessoas com AVC podem melhorar a saúde mental e reduzir a sobrecarga do cuidador. No entanto, não devemos nos esquecer de que há uma interação entre todos os aspectos da sobrecarga (saúde física e mental, dificuldades financeiras e rede de suporte social pequena) ${ }^{(10)}$.

Esse mesmo autor recomenda a utilização dos benefícios do processo de grupo, oferecidos, geralmente, por associações de pacientes (Associação de Pessoas com AVC) como suporte durante o período de adaptação e no enfrentamento de novos eventos que forem surgindo. Essa estratégia pode ajudar a reduzir as consultas e a institucionalização tanto do paciente como do cuidador.

Os fatores que estão relacionados com a diminuição da sobrecarga são: maior nível de escolaridade, incapacidades físicas moderadas e rendas financeiras $\operatorname{altas}^{(10)}$

\section{CONSIDERAÇÕES FINAIS}

Este artigo traz uma fundamentação teórica acerca das conseqüências psicossociais sobre cuidadores familiares de pessoas com AVC, ao vivenciarem as suas experiências no desempenho do papel.

O trabalho reúne variáveis importantes que emergiram a partir de pesquisas publicadas em periódicos nas décadas de 80 e 90, relacionadas à sobrecarga de cuidadores familiares de pessoas com AVC.

Acredito que a divulgação deste conhecimento possa se constituir num referencial à equipe de saúde durante a elaboração de intervenções direcionadas às famílias afetadas por tal acometimento, como estratégia de melhoria da qualidade de vida das mesmas.

Ademais, é necessário que se realizem investigações por meio de abordagens qualitativas, visando a ampliação do conhecimento sobre os eventos que compõem os fenômenos, a partir das experiências dos sujeitos. 


\section{REFERÊNCIAS BIBLIOGRÁFICAS}

1. Bocchi SCM. O familiar cuidador da pessoa com AVC: uma análise do conhecimento. Botucatu: UNESP-Faculdade de Medicina; 2000.

2. Schene AH. Objective and subjective dimensions of family burden: towards and integrative framework for research. Soc Psychiatry Psychiatr Epidemiol 1990 Nov; 25(6):289-97.

3. Platt S. Measuring the burden of psychiatric illness on the family: an evaluation of some rating scales. Psychol Med 1985 May; 15(2):383-93.

4. Bardin L. Análise de conteúdo. Lisboa: Edições 70; 1977.

5. Minayo MCS. O desafio do conhecimento: pesquisa qualitativa. São Paulo (SP): HUCITEC-ABRASCO; 1992.

\section{REFERÊNCIAS BIBLIOGRÁFICAS: CORPUS DE ANÁLISE}

6. Coughlan AK, Humphrey M. Presenile stroke: long-term outcome for patients and their families. Rheumatol Rehabil 1982 May; 21(2):115-22.

7. Silliman RA, Fletcher RH, Earp JL, Wagner EH. Families of elderly stroke: effects of elderly stroke patients: effects of home care. J Am Geriatr Soc 1986 Sept; 34(9):643-8.

8. Williams SE, Freer CA. Aphasia: its effect on marital relationships. Arch Phys Med Reabil 1986 Apr; 67(4):250-2.

9. Bronstein KS Psychosocial components in stroke: implications for adaptation Nurs Clin North Am 1991 Dec; 26(4):1007-17.

10. Printz-Feddersen V. Group process effect on caregiver burden. J Neurosc Nurs 1990 Jun; 22(3):164-8.

11. Cassidy TP, Gray C. Stroke and the carer. Br J Gen Pract 1991 Jul; 41(348):267-8.

12. Béthoux F, Calmels P, Gautheron V, Minaire P. Quality of life of the spouses of stroke patients: a preliminary study. Int $J$ Rehabil Res 1996 Dec; 19(4):291-9.

13. Grant JS. Home care problems experienced by stroke survivors and their family caregivers. Home Healthc Nurse 1996 Nov; 14(11):892-902.

14. Anderson CS, Linto J, Stewart-Wynne EG A populationbased assessment of the impact and burden of caregiving for long-term stroke survivors. Stroke 1995 May; 26(5):843-9. 15. Dennis M, O'Rourke S, Lewis S, Sharpe M, Warlow C A quantitative study of the emotional outcome of people caring for stroke survivors. Stroke 1998 Sept; 29(9):1867-72.

16. Periard ME, Ames BD. Lifestyle changes and coping patterns among caregivers of stroke survivors. Public Health Nurs 1993 Dec; 10(4):252-6.

17. Holbrook M. Stroke: social and emotional outcome. J R Coll Physicians Lond 1982 Apr; 16(2):100-4.

18. Carnwath TCM, Johnson DAW. Psychiatric morbidy among spouses of patients with stroke. Br Med J 1987 Jan; 294(6569):409-11.

19. Robinson-Smith G, Maboney C. Coping and marital equilibrium after stroke. J Neurosc Nurs 1995 Apr; 27(2):839.

20. Silliman RA, Fletcher RH, EARP J, Wagner EH. Families of elderly stroke: effects of elderly stroke patients: effects of home care. JAGS 1986 Sept; 34(9):643-8.
21. Williams A. What bothers caregivers of stroke victims? J Neurosc Nurs 1994 Jun; 26(3):155-61.

22. Field D, Cordle CJ, Bowman, GS. Coping with stroke at home. Int Reabil Med 1983;5(2):96-100.

23. McLean J, Roper-Hall A, Mayer P, Main A. Service needs of stroke survivors and their informal carers: a pilot study. J Adv Nurs 1991 May; 16(5):559-64.

24. Williams AM. Caregivers of person with stroke: their physical and emotional well being. Qual Life Res 1993 Jun; 2(3):213-20.

25. Draper BM, Poulos CJ, Cole AMD, Poulos RG, Ehrlich FE. A comparison of caregivers for elderly stroke and dementia victims. J Am Geriatr Soc 1992 Sept; 40(9):896-901. 